\title{
Candidate Centaurs trapped in mean motion resonances with Uranus
}

\author{
Y. Masaki^ and H. Kinoshita \\ National Astronomical Observatory, Japan, 2-21-1, Osawa, Mitaka, Tokyo 181-8588, Japan \\ Received 10 September 2002 / Accepted 5 March 2003

\begin{abstract}
We investigated the dynamical evolution of Centaurs moving in the region of the giant planets. Perturbations from four major planets (Jupiter, Saturn, Uranus and Neptune) were included. We have found that $2000 \mathrm{QC}_{243}$ is trapped in a 5:4 mean motion resonance with Uranus and $2001 \mathrm{XZ}_{255}$ is also locked in a 4:3 resonance. Both Centaurs are moving between the orbits of Saturn and Uranus. They leave the resonances after a few hundred thousand years. Some other Centaurs (10199 Chariklo and 1994TA) also show the resonant character. Their orbital elements (except for Chariklo) are not well determined. However, the region between Saturn and Uranus may play the role of a temporary reservoir for Centaurs.
\end{abstract}

Key words. celestial mechanics - minor planets, asteroids

\section{Introduction}

For the past several years, automated observational systems operated all over the world have detected a tremendous number of asteroids and revealed their orbital nature. As of August 2002, 40000 or more objects had been registered as "numbered asteroids", and more than a hundred thousand bodies remain unnumbered.

The increase in orbital information enables us to depict the distribution of minor bodies in the Solar system. Most cataloged objects classified as asteroids populate the region between Mars and Jupiter, forming the "main belt". Since the 1990s, another type of small objects has also been detected in the far-Neptunian region. These are called Edgeworth-Kuiper belt objects (EKBOs).

The dynamics of main belt asteroids (MBAs) and EKBOs have been intensively studied by many astronomers. We can see some dynamical structures in the distribution of these objects. Mean motion resonances with Jupiter (for MBAs) or Neptune (for EKBOs) make eminent gaps or groups.

However, some minor objects, classified as Centaurs, are distributed in the region of the giant planets (between Jupiter and Neptune). We can only find some tens of objects with the semimajor axis $a$ of $6<a<30 \mathrm{AU}$, including unnumbered objects. (2060) Chiron is the first detected body in this region and shows cometary activity (e.g. Tholen et al. 1988). Due to its high eccentricity, its orbital evolution shows a chaotic nature

Send offprint requests to: Y. Masaki, e-mail: ymasaki@gsi.go.jp

* Present address: Space Geodesy Research Division, Geographical Survey Institute, 1, Kitasato, Tsukuba, Ibaraki, 305-0811, Japan.
(Oikawa \& Everhart 1979). The discoveries of other Centaurs have stimulated the studies of their dynamical evolution and lifetimes (e.g. Asher et al. 1994; Dones et al. 1996). Today, it is believed that Centaurs are in the transitional state from EKBOs to short periodic comets.

The stability of the planetesimals in the giant planets region has been studied by several authors. Duncan et al. (1989) simplified the problem by assuming that planetesimals, placed in circular orbits initially, are perturbed by only two nearby planets. They concluded that planetesimals in the Saturn-Uranus region can survive for the lifetime of the Solar system.

However, the following studies led to negative results: Gladman \& Duncan (1990) studied the stability of test particles, placed initially in circular orbits, under the perturbations of four giant planets. They concluded that all particles placed between Saturn and Uranus became unstable in the timescale of millions of years. Holman \& Wisdom (1993) observed that most test particles placed between Saturn and Uranus are removed in 10 Myr. Grazier et al. (1999) vigorously investigated the lifetime of planetesimals placed in elliptical, inclined orbits. They came to the conclusion that particles are removed within the timescale of 1 to $10 \mathrm{Myr}$. Particles with some particular semimajor axes (e.g. $a=12.5,14.4,16.0 \mathrm{AU}$ ) can survive for a much longer time than others. Holman (1997) discussed the lifetime of test particles placed in the Uranus-Neptune region and found that two regions $(a \sim 24.6$ and 25.6 AU) harbour the long-lived test particles.

In this study, we integrated the equations of motion of 44 numbered and unnumbered Centaurs in the giant planets region. We have found that some Centaurs are temporarily locked in a mean motion resonance with Uranus. For the main 
belt asteroids, similar numerical simulations have already been reported (e.g. Kozai \& Nakai 2002).

Centaurs $2000 \mathrm{QC}_{243}$ and $2001 \mathrm{XZ}_{255}$ have the semimajor axes of $\sim 16 \mathrm{AU}$, located in the region between Saturn and Uranus. Both have low orbital eccentricities $(e \sim 0.2$ and $e \sim 0.05$ ), in contrast to other Centaur objects (e.g. $e \sim 0.7$ ). We have found that critical arguments librate around 0 degrees for several hundred thousand years. The Centaurs (10199) Chariklo and 1994TA also show the resonant character.

We report the results in the following sections.

\section{Methods of analysis}

Now we consider the motion of Centaurs around the Sun under the perturbations of the four giant planets (Jupiter, Saturn, Uranus and Neptune). We treat Centaurs as massless particles.

The orbital data of Centaurs are obtained through the internet from the MPCORB database maintained by The IAU Minor Planet Center ${ }^{1}$. Additional data on the accuracy of the ephemeris are also available from this database. Positions and velocities of the disturbing planets at the epoch of the MPCORB data are obtained from the DE405 ephemeris supplied by JPL.

We integrated equations of motion by extrapolation (Bulirsch-Stoer) code for $1 \mathrm{Myr}$ forward and backward. We adopted heliocentric coordinates and an invariable plane based on the four planets as the reference plane for outputting results. We selected 44 Centaurs whose osculating semimajor axis at the epoch lies in the range of $6<a<35$ AU for our calculation.

In advance, we monitored the orbital elements (especially the semimajor axis) of each Centaur to judge whether it is likely to be in a resonant state or not. Next, we checked the critical argument appropriate for the resonance.

\section{Results}

We have found that $2000 \mathrm{QC}_{243}$ and $2001 \mathrm{XZ}_{255}$ are temporarily locked in a mean motion resonance with Uranus at present. We also see the resonant character in (10199) Chariklo and 1994TA, but for a shorter duration. Their orbital elements are listed in Table 1.

The evolution of the osculating elements of the first two Centaurs is shown in Fig. 1. We extracted data in the range of $0.1 \mathrm{Myr}$ forward and backward for the plotting. The bottom panels of the figures show the critical arguments: $\sigma=$ $5 \lambda_{\mathrm{U}}-4 \lambda-\varpi$ for $2000 \mathrm{QC}_{243}$ and $\sigma=4 \lambda_{\mathrm{U}}-3 \lambda-\varpi$ for $2001 \mathrm{XZ}_{255}$, where $\lambda$ and $\varpi$ are the mean longitude and the perihelion longitude of the Centaurs and $\lambda_{\mathrm{U}}$ is the mean longitude of Uranus.

The semimajor axes for these resonances obtained from Kepler's third law are 16.6 AU (for the 5:4 resonance) and 15.9 AU (for the 4:3). The osculating elements listed in Table 1 are slightly different from these values, but this difference is due to the resonant libration.

\footnotetext{
1 The URL is http://cfa-www.harvard.edu/iau/mpc.html.
}

Since the critical arguments exhibit librations around 0 degrees, both Centaurs are trapped in a first order $(p:(p-1))$ mean motion resonance. Therefore, longitudinal conjunctions between the Centaur and Uranus occur around the perihelion passage of the Centaur, to avoid a close approach to Uranus.

However, neither can stay in the resonances eternally. They leave this resonant state after $\sim 0.1 \mathrm{Myr}$ for $2001 \mathrm{XZ}_{255}$ and $\sim 0.8 \mathrm{Myr}$ for $2000 \mathrm{QC}_{243}$. These two Centaurs are temporarily locked in these resonances.

Due to the secular or longer periodic perturbations from the nearby giant planets, their orbits are successively changed. For a longer timespan (such as the period of the apsidal motion; the order of $10^{5}$ years), their orbital eccentricities increase irregularly. After these Centaurs leave the resonance, the protection mechanism against the approach to Uranus also collapses. Successive large perturbations from Uranus accelerate their orbital evolution. Finally, their orbits become planet (Saturn or Uranus)-crossing. After leaving the resonances, their orbital evolution shows chaotic behavior.

Evolutional tracks of these orbits on an $(a, e)$ plane (the semimajor axis versus the eccentricity) are shown in Fig. 2. The orbit of $2000 \mathrm{QC}_{243}$ has evolved from a Saturn-crossing one to its present state. After the orbit leaves the resonant state, Uranian perturbations strongly affect its evolution. For $2001 \mathrm{XZ}_{255}$, the orbital eccentricity decreases over the past few hundred thousand years, locked in a resonant state. After a collapse of the resonant mechanism, the close approaches to Uranus accelerate its orbital evolution and the orbit plunges into a chaotic track.

However, due to uncertainties of the provisional orbital data and chaotic orbital evolution caused by repeated close approaches to the giant planets, these evolutional tracks do NOT perfectly depict their evolutional tracks.

Some other Centaurs also show a resonant character, but for shorter durations. The numbered Centaur (10199) Chariklo had locked in a 4:3 resonance in the past (see Fig. 3). The critical argument $\sigma=4 \lambda_{\mathrm{U}}-3 \lambda-\varpi$ is librating around 0 degree. 1994TA is in an 11:9 resonance (the semimajor axis is $\sim 16.8 \mathrm{AU}$ ) at present. Interestingly, the critical argument contains the longitude of the perihelion of Uranus $\varpi_{\mathrm{U}}$; i.e. $\sigma=11 \lambda_{\mathrm{U}}-9 \lambda-2 \varpi_{\mathrm{U}}$. It librates around 180 degrees. The resonant state only lasts for a few tens of thousands of years.

\section{Clone tests}

Up to August 2002, orbital elements of $2000 \mathrm{QC}_{243}$ and $2001 \mathrm{XZ}_{255}$ are NOT well-determined because observational arcs are too short to permit their determining (see Table 1). Especially, the elements of $2001 \mathrm{XZ}_{255}$ are derived from only one oppositional season of observation, and they contain orbital fitting errors. After more observations are made, their elements may change from the current provisional values.

Hereafter, we assess the tolerance to the change in the present orbital elements to keep the system in a resonant state.

We introduce some sets of "clones" whose semimajor axis and eccentricity are changed by a small amount from the MPC data. We numerically integrated for 50000 years and monitored the critical argument whether a clone is in the resonance or not. 
Table 1. Osculating elements of Centaurs 2000QC $243,2001 \mathrm{XZ}_{255}$, (10199) Chariklo and 1994TA. The original data are listed in The MPCORB database (Version: Aug. 18, 2002) maintained by The IAU Minor Planet Center. See also http://cfa-www.harvard.edu/iau/info/MPOrbitFormat.html for further explanations of each item.

\begin{tabular}{lrrrr}
\hline \hline Item & $2000 \mathrm{QC}_{243}$ & ${ }^{2001 \mathrm{XZ}_{255}}$ & $(10199)$ & $1994 \mathrm{TA}$ \\
\hline Semimajor axis $a[\mathrm{AU}]$ & 16.4958326 & 16.0429096 & 15.8285197 & 16.8097671 \\
Eccentricity $e$ & 0.2016333 & 0.0438819 & 0.1738749 & 0.2996731 \\
Inclination $I[\mathrm{deg}]$ & 20.77197 & 2.60700 & 23.36552 & 5.39557 \\
Longitude of the ascending node $\Omega[\mathrm{deg}]$ & 337.84861 & 77.81235 & 300.39518 & 137.75032 \\
Argument of the perihelion $\omega[\mathrm{deg}]$ & 152.51174 & 294.46211 & 241.33396 & 154.36691 \\
Mean anomaly at the epoch $l_{0}[\mathrm{deg}]$ & 222.34865 & 87.69243 & 351.03363 & 92.42874 \\
\hline Epoch [JD] & 2452400.50 & 2452400.50 & 2452400.50 & 2452400.50 \\
\hline Number of oppositions & 3 & 1 & 5 & 5 \\
Uncertainty parameter & 3 & 6 & 2 & 2 \\
rms residual [arcsec] & 0.44 & 0.15 & 0.50 & 0.65 \\
\hline
\end{tabular}
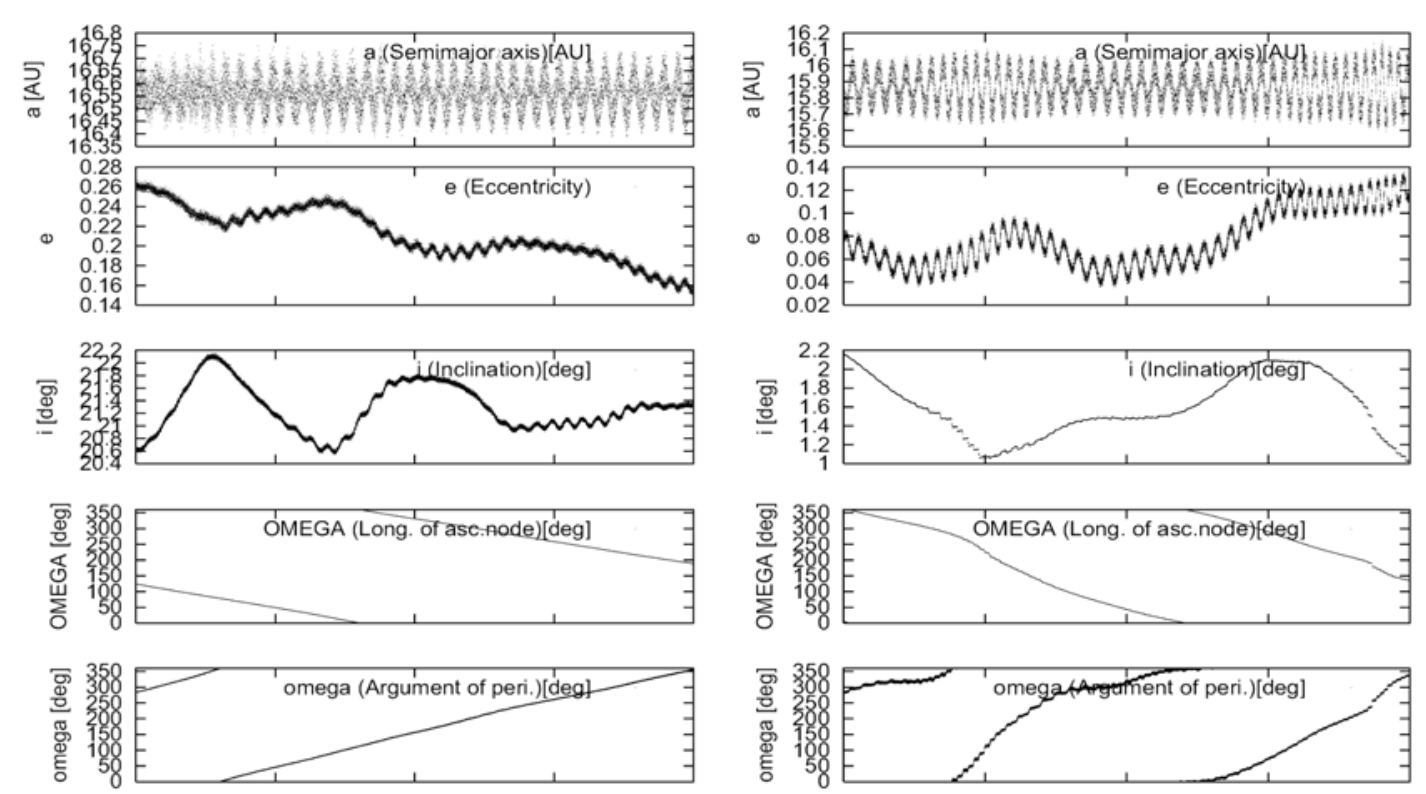

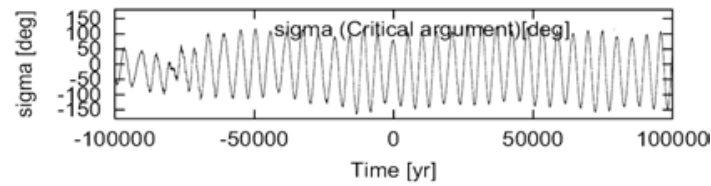

(a) $2000 \mathrm{QC}_{243}$

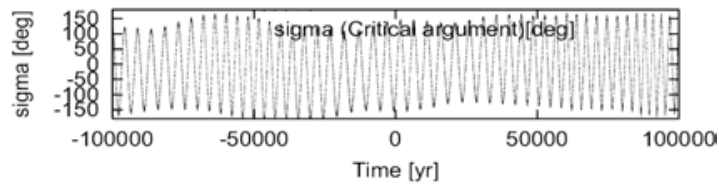

(b) $2001 \mathrm{XZ}_{255}$

Fig. 1. Numerically integrated orbital elements of a) $2000 \mathrm{QC}_{243}$ and b) $2001 \mathrm{XZ}_{255}$. The bottom panels show the critical arguments: $\sigma=$ $5 \lambda_{\mathrm{U}}-4 \lambda-\varpi$ for $2000 \mathrm{Q} C_{243}$ and $\sigma=4 \lambda_{\mathrm{U}}-3 \lambda-\varpi$ for $2001 \mathrm{XZ}_{255}$. They librate around 0 degrees.

The results, plotted on a $(t, \sigma)$ plane (the time versus the critical argument), are shown in Fig. 4 a for $2000 \mathrm{QC}_{243}$ and Fig. $4 \mathrm{~b}$ for $2001 \mathrm{XZ}_{255}$. Each panel corresponds to one clone; the horizontal axis spans from 0 to 50000 years ( 10000 years per grid) and the vertical axis spans from -180 to 180 degrees ( 90 degrees per grid).

The results show that even if the semimajor axis is shifted by $\Delta a=+0.05 \mathrm{AU}$ for $2000 \mathrm{QC}_{243}$, the resonant character still holds for at least 50000 years. The initial eccentricity is larger by $\Delta e=+0.04$ than the current value, and the system stays in the resonance. For $2001 \mathrm{XZ}_{255}$, a smaller semimajor axis is preferred for the system holding the resonant state.
The resonant nature is not sensitive to the change in the eccentricity. However, these integrations also indicate that if the current provisional elements contain large uncertainties, these Centaurs do not librate in the mean motion resonances.

We also check the effect of ambiguity in the argument of the perihelion because, for a nearly circular orbit, the perihelion is hardly determined. Therefore, we shift the value by a small amount and monitor the critical argument. (Generally speaking, the longitude of the celestial body is well-determined even in the case of a circular orbit. Therefore, we adjust the mean anomaly at the initial epoch so as not to change the initial mean longitude when we shift the argument of the perihelion). 


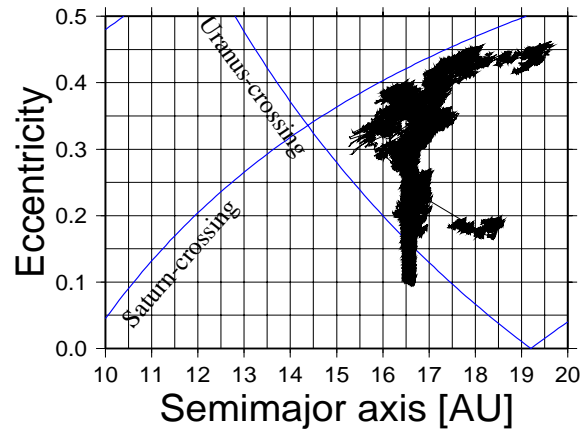

(a) $2000 \mathrm{QC}_{243}$

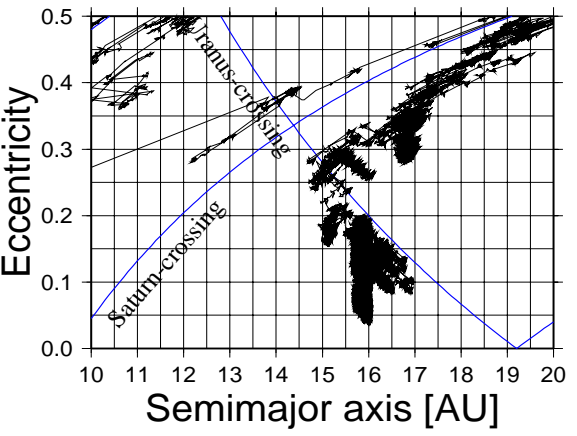

(b) $2001 \mathrm{XZ}_{255}$

Fig. 2. Orbital evolution on an $(a, e)$ plane. The plotted data cover from -1 Myr to +1 Myr. For convenience, the planet-crossing curves are also shown. For drawing these curves, we assume that the Centaur and giant planets are moving on a common plane and the planetary eccentricities are fixed to the present values.
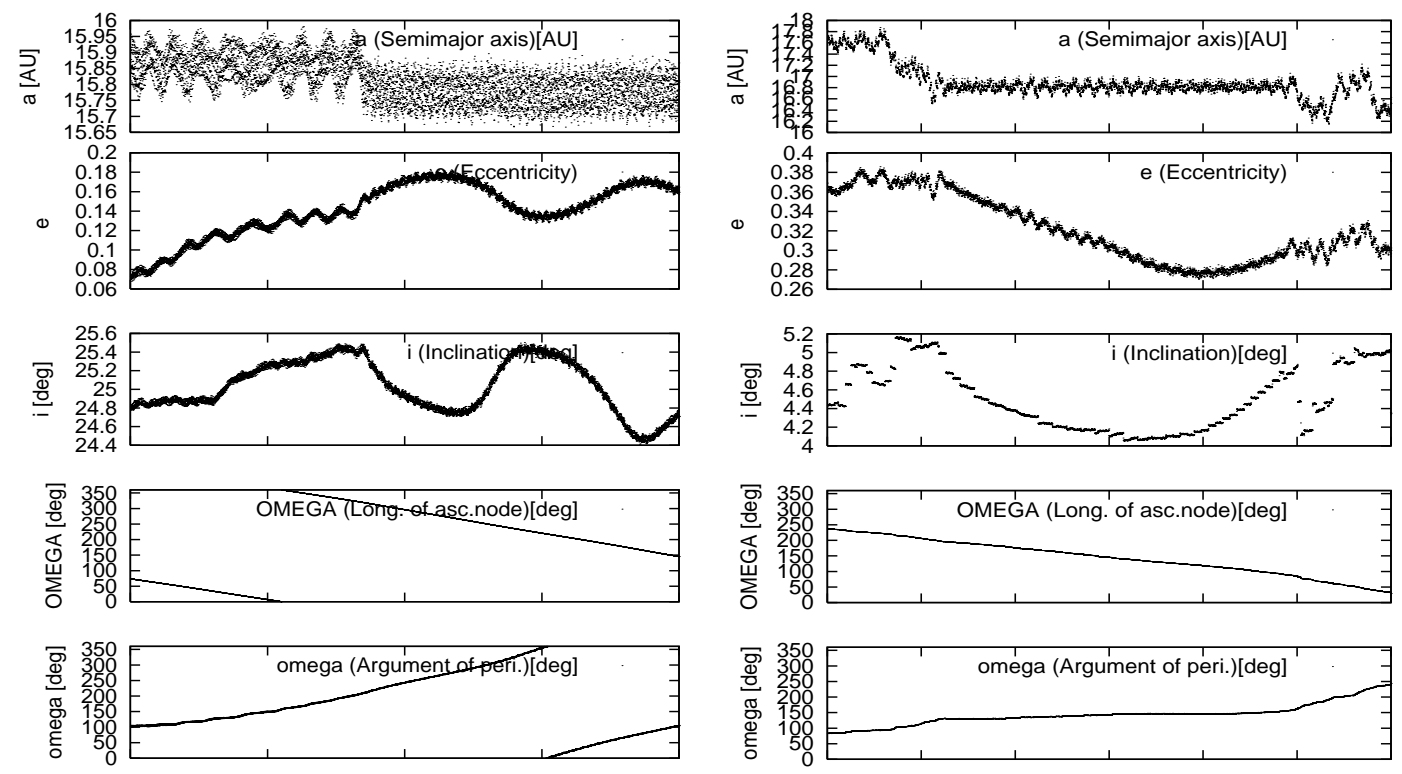

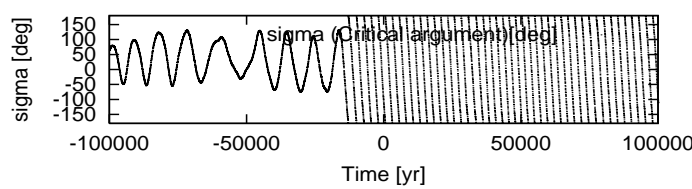

(a) (10199) Chariklo

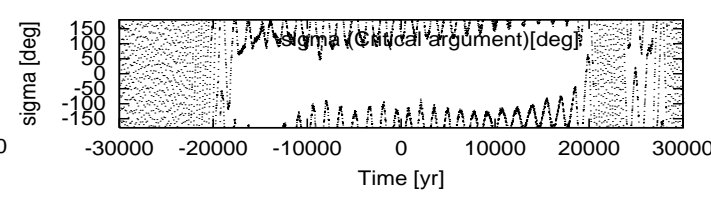

(b) $1994 \mathrm{TA}(-30 \mathrm{kyr}$ to $+30 \mathrm{kyr})$

Fig. 3. Numerically integrated orbital elements of a) (10199) Chariklo and b) 1994TA ( $-30 \mathrm{kyr}$ to $+30 \mathrm{kyr})$. The bottom panels show the critical arguments of $\sigma=4 \lambda_{\mathrm{U}}-3 \lambda-\varpi$ for (10199) Chariklo and $\sigma=11 \lambda_{\mathrm{U}}-9 \lambda-2 \varpi_{\mathrm{U}}$ for 1994TA. (10199) Chariklo is not in the resonance at present.

We confirmed that the resonant nature is not sensitive to small errors in the argument of the perihelion.

\section{Discussion}

Do other faint and unknown Centaurs stay around the region between Saturn and Uranus? Does the resonance play the role of a (temporary) reservoir for some Centaurs?

Since most Centaurs have highly eccentric and/or planetcrossing orbits, their orbits are rapidly changed by strong perturbations from giant planets. However, a slightly eccentric orbit in the Saturn-Uranus region shows slower orbital evolution than a highly eccentric one, even if it has a relatively low orbital inclination. This region may temporarily harbour some Centaurs until long periodic or secular perturbations expel them.

Today, several Centaurs are seen in this region (Fig. 5). None of them (except for 1994TA) drastically change their semimajor axes for the next 50000 years. These loweccentricity Centaurs make a clear contrast with most other Centaurs; the latter group has a highly eccentric or planetcrossing orbit and changes its orbit within a shorter timescale.

We note that the orbital eccentricity is suppressed at a small value while a Centaur is trapped in a mean motion resonance. 


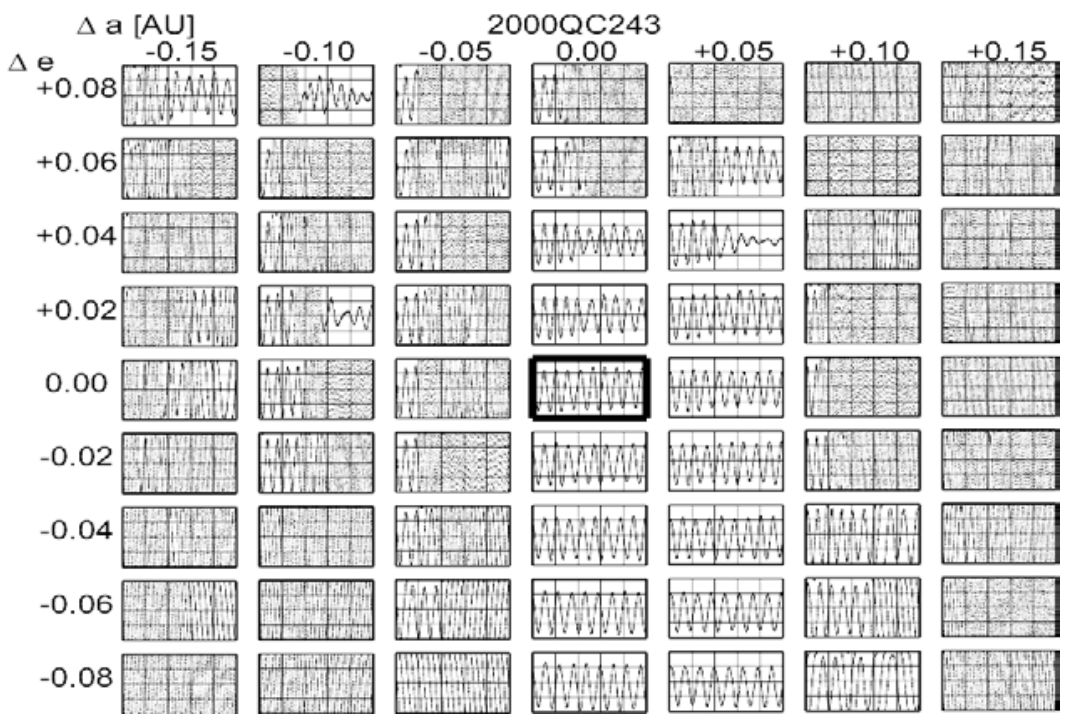

(a) $2000 \mathrm{QC}_{243}$

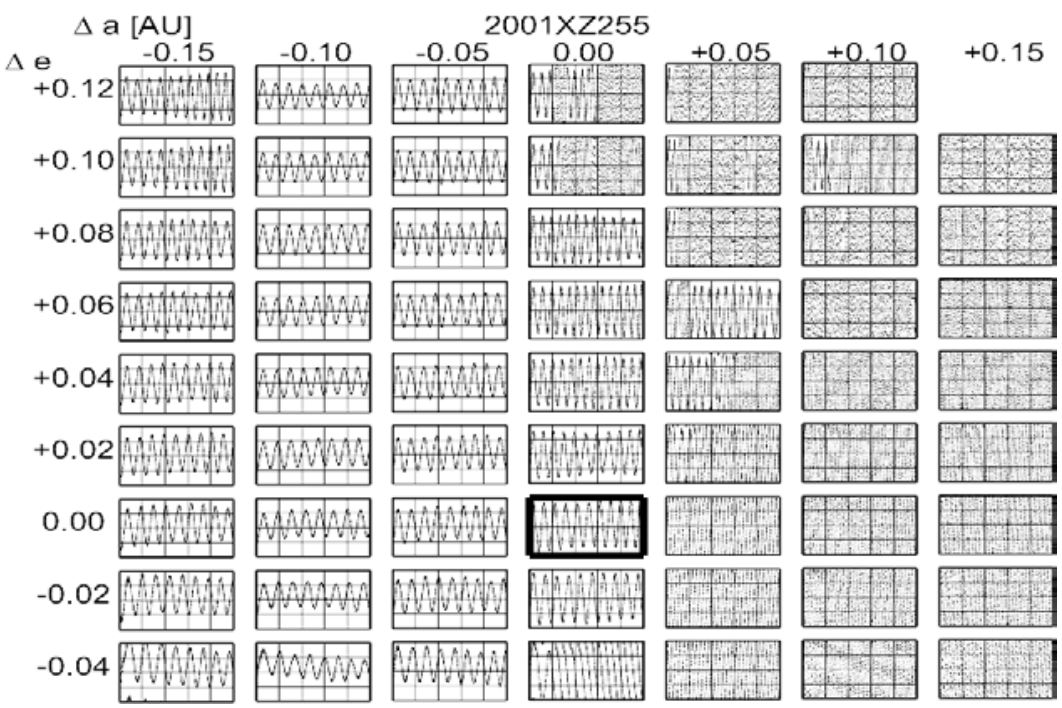

(b) $2001 \mathrm{XZ}_{255}$

Fig. 4. Clone tests for a) $2000 \mathrm{QC}_{243}$ and b) $2001 \mathrm{XZ}_{255}$. Each panel corresponds to one clone particle. The initial orbital elements are shifted by small amounts designated as $\Delta a \mathrm{AU}$ and $\Delta e$ at the top and the left of the figures. The time-evolution of the critical argument is plotted on a $(t, \sigma)$ plane; the horizontal axis spans from 0 to 50000 years (one division equals 10000 years) and the vertical axis spans from -180 to 180 degrees (one division equals 90 degrees). The result of the original orbital data listed in Table 1 is plotted on a panel with a thick frame which is located at $\Delta a=0.00 \mathrm{AU}$ and $\Delta e=0.00$.

Similar phenomena are also reported in Levison \& Duncan (1997) for some resonant EKBOs.

The existence of resonant Centaurs indicates that some long-lived objects may stay in the region of $a \sim 16 \mathrm{AU}$. It is expected that the mean motion resonance with Uranus contributes to the temporary stability.

\section{Conclusion}

We integrated the equations of motion of 44 Centaurs. Four giant planets are included in the calculation. We have found that Centaurs $2000 \mathrm{QC}_{243}$ and $2001 \mathrm{XZ}_{255}$ are likely to be temporarily locked in mean motion resonances with Uranus. Some other Centaurs also show a resonant character. These objects move in low eccentricity orbits, which contrast with the other Centaurs. They do not show significant changes in the orbital elements for at least some tens of thousands of years. They are protected from close approaches to the giant planets due to their small eccentricities and the resonant mechanism.

However, since the orbits of the resonant Centaurs are strongly perturbed by giant planets, their eccentricities are gradually pumped up to collapse the resonant state. Finally, their orbits evolve into Saturn/Uranus-crossing orbits.

We interpret this situation to mean that these Centaurs are temporarily harboured in this region. It is consistent with previous studies (Gladman \& Duncan 1990; Holman \& Wisdom 1993; Grazier et al. 1999; see Sect. 1) on the stability of planetesimal distributed in the giant planets region. The mean 


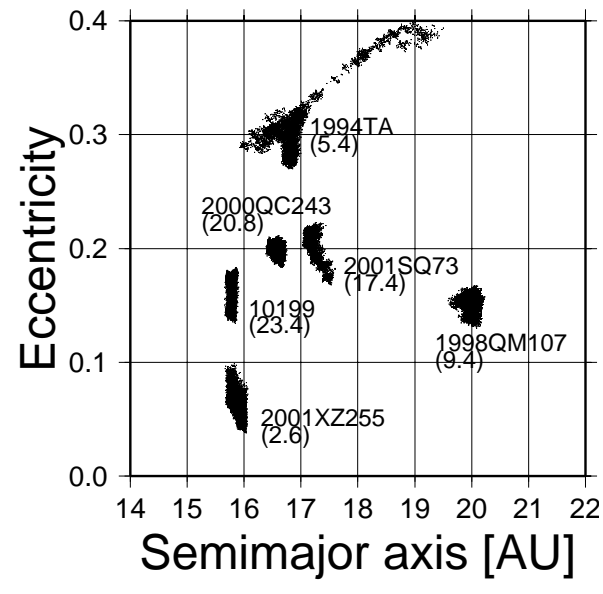

Fig. 5. Orbital evolution of other Centaurs near the resonant candidates. We plot the evolutional tracks on an $(a, e)$ plane for the next 50000 years. These objects are not wandering around so much on the $(a, e)$ plane, in contrast to other Centaurs which have eccentric and/or planet-crossing orbits. Large orbital changes in the elements of 1994TA occur after the resonance is unlocked. The present orbital inclinations (unit: degree) are also indicated in parentheses.

motion resonance with Uranus contributes to the temporary stability in this region.

When we discuss the material evolution of Centaurs, it is also important to think about the timescale of their dynamical evolution because it is believed that the past environment (e.g. thermal history) and their ages are recorded on the surface material. The dynamical character may provide some hints about the nature of Centaurs.

Acknowledgements. We appreciate a referee's suggestions and improvements of the original manuscript. We express our sincere thanks to Ms. Yolande McLean for improving our English. We are also grateful to Mr. Hiroshi Nakai, at National Astronomical Observatory, Japan, for giving us the information on the asteroidal database.

\section{References}

Asher, D. J., Bailey, M. E., Hahn, G., \& Steel, D. I. 1994, MNRAS, 267, 26

Dones, L., Levison, H. F., \& Duncan, M. 1996, in Completing the Inventory of the Solar System, ed. T. W. Rettig, \& J. M. Hahn, ASP Conf. Ser., 107, 233

Duncan, M., Quinn, T., \& Tremaine, S. 1989, Icarus, 82, 402

Gladman, B., \& Duncan, M. 1990, AJ, 100, 1680

Grazier, K. R., Newman, W. I., Varadi, F., Kaula, W. M., \& Hyman, J. M. 1999, Icarus, 140, 353

Holman, M. J. 1997, Nature, 387, 785

Holman, M. J., \& Wisdom, J. 1993, AJ, 105, 1987

Kozai, Y., \& Nakai, H. 2002, preprint

Levison, H. F., \& Duncan, M. J. 1997, Icarus, 127, 13

Oikawa, S., \& Everhart, E. 1979, AJ, 84, 134

Tholen, D. J., Hartmann, W. K., \& Cruikshank, D. P. 1988, IAU Circ. 4554 